\title{
La financiación por medio del crowdfunding del audiovisual: el caso del cortometraje Juan y la nube
}

\author{
Carlota CORONADO RUIZ \\ carlotacoronado@gmail.com \\ Julio LARRAÑAGA RUBIO \\ julio.larranaga@ccinf.ucm.es \\ Universidad Complutense de Madrid
}

Recibido: 15/06/2015

Aceptado: 21/09/2015

\section{RESUMEN}

En el artículo se analizan las posibilidades que tiene el crowfunding (CF) para financiar el audiovisual. Se explica el modelo de financiación del CF y se indican las distintas ventajas que reporta para el promotor este modelo de financiación alternativa. Además se exponen ampliamente las principales características del proceso de CF seguido para financiar el cortometraje Juan y la nube (que se alzó con el Goya al Mejor corto de animación en el año 2015). Se comentan también los principales motivos que llevan a los mecenas a financiar los proyectos

Palabras claves: crowfunding, financiación alternativa, películas, cortometrajes, mecenas, recompensas.

Financing audiovisuals through crowdfunding: the case of Juan y la nube

\begin{abstract}
This article studies the possibilities of financing audiovisuals through crowdfunding (CF). The model of financing by CF is described as well as the different advantages that this alternative model of financing provides promotors. The main features of the chosen CF procedure to finance the short film Juan y la nube (that was awarded with the Goya prize to the best entertainment short film of 2015) will also be described in length. The main reasons that lead sponsors to finance projects are also subject to comment. Keywords: crowdfunding, alternative financing, films, short films, sponsors, rewards.
\end{abstract}

\section{Referencia normalizada}

Coronado Ruiz, C. y Larrañaga Rubio, J. (2015). "La financiación por medio del crowdfunding del audiovisual: el caso del cortometraje Juan y la nube”. Documentación de las Ciencias de la Información, Vol. 38: páginas 203-221 


\section{INTRODUCCIÓN}

En el artículo se analizan las posibilidades que tiene el crowfunding (CF) para financiar el audiovisual. En primer lugar, se expone utilizando los datos proporcionados por varios informes, la situación del audiovisual en España, especialmente la situación de las películas. A continuación se explica el modelo de financiación del CF, indicando los diferentes tipos que existen así como las cifras de negocio que representa tanto en España como en el ámbito europeo. Se mencionan también las distintas ventajas que reporta para el promotor este modelo de financiación. En el siguiente punto se exponen de una forma amplia las principales características del proceso de CF seguido para financiar el cortometraje Juan y la nube (que se alzó con el Goya al Mejor corto de animación en el año 2015). Por último, se analizan los principales motivos que llevan a que los mecenas españoles participen en los proyectos de CF.

\section{EL CROWDFUNDING}

El cine producido en España obtuvo una recaudación durante el año 2013 (último año para el que hay información disponible) de 70,15 millones de euros, con 11,01 millones de espectadores, según la información que proporciona el "Boletín Informativo de Cine. Año 2013” del Instituto de la Cinematografía y de las Artes Audiovisuales (ICAA). Dichas cifras de recaudación y del número de espectadores son las más reducidos de los últimos 10 años (ICAA, 2014:15 y 16) y han llevado a que la cuota del cine español en el año 2013 (expresada como el porcentaje que supone su recaudación en relación a la cifra de la recaudación total) únicamente sea del 13,86\%.

Por el contrario, se produce una sensible mejora en la demanda del cine español en el año 2014, ya que se incrementa su cuota hasta el 24,9\% consiguiendo 20,8 millones de espectadores según muestran las previsiones realizadas por José María Álvarez Monzoncillo y Javier López Villanueva en su aportación "El Audiovisual Español: evolución en curso" incluida en el "II Informe sobre el estado de la cultura en España 2914: la salida digital (ICE-2014)" coordinado por Enrique Bustamante y Fernando Rueda. Aunque estos buenos resultados deben ser matizados porque una gran parte de la demanda se concentra en "cuatro películas: 8 apellidos vascos (con cerca del 50\% de la recaudación total del cine español), El Niño, La isla mínima y Torrente” (Álvarez y López, 2015: 67). Además, es preocupante el elevado número de películas españolas que ni siquiera se llegan a estrenar en los cines, cerca del $25 \%$.

Desde el punto de vista de la oferta, el sector atraviesa una grave crisis, como se puede observar de forma inmediata al comparar las cifras de financiación existentes antes de la crisis económica con la actuales, pues la "la financiación se ha dividido por dos, lo que se ha traducido hasta 2013, en menos películas rodadas con presupuestos cercenados” (Álvarez y López, 2015: 66). Esta disminución se debe a dos motivos. En primer lugar a que la fuerte reducción sufrida por los ingresos publicitarios y las subvenciones ha conllevado que "las televisiones públicas y privadas han 
reducido a la mitad sus inversiones cinematográficas ... En concreto, en 2011 y 2012 las operadoras destinaron 98,8 y 75,8 millones de euros al cine en español, respectivamente" (Álvarez y López, 2015: 67). Aunque de estos ingresos únicamente "depende una pequeña élite del cine español, en torno a un 5 por 100 de los films, con fuertes inversiones del productor y de los canales de televisión, además de importantes ingresos provenientes de las preventas al extranjero" (Álvarez y López, 2015: 69), estas películas determinan la cuota de mercado del cine español. Además, la reducción de ingresos de las televisiones también ha conllevado una reducción en la producción de ficción televisiva.

También la caída de las subvenciones del ICAA, contribuyó a la reducción de la oferta. Las subvenciones del Ministerio de Educación, Cultura y Deporte, tuvieron una reducción desde los 92,4 millones de euros del año 2010 hasta 36,9 en el 2013. Se comenta en el informe del ICAA, anteriormente mencionado, que la mayor parte de este importe se destinó al sector de la producción "por considerar que es el motor inicial de la actividad cinematográfica, el de mayor riesgo y el de inversiones más voluminosas" (ICAA, 2014:17). También de esta cantidad se destinan 5 millones de euros para ayudas sobre proyecto para "la producción de películas de largometraje de nuevos realizadores, documentales o películas que posean un valor cinematográfico, cultural o social, ratifican el apoyo a la incorporación de nuevos talentos” (ICAA, 2014:17). Se ayuda a 14 proyectos de ficción, 11 documentales y 2 películas de animación.

Además, las Comunidades Autónomas también han reducido sus subvenciones: "lo mismo ha pasado con las Comunidades Autónomas, pues han reducido sus subvenciones anuales de 40 a 20 millones de euros” (Álvarez y López, 2015:67).

La dura situación que atraviesa actualmente el audiovisual español lleva a que se afirme que "el cine y la ficción televisiva han entrado en una espiral de crisis estructural que será difícil recuperar en años venideros” (Álvarez y López, 2015:74). Estos autores especifican que es necesario intentar recuperar los niveles de producción del audiovisual anteriores a la crisis económica y para ello "bastaría con no dejar a las televisiones públicas atrapadas en el mantenimiento de sus costes fijos y con vertebrar una política cinematográfica pensando en el desarrollo de las nuevas tecnologías y en el fomento de la distribución en la red” (Álvarez y López, 2015:74).

En el informe de la ICAA también se incide en la situación que atraviesa el sector de la producción en nuestro país y se establece la necesidad de fortalecer su estructura empresarial para que de esta forma se pueda conseguir una adecuada solvencia financiera. Además se afirma que la mayoría del sector mantiene una escasa actividad poniéndose como ejemplo de ello que de las 267 empresas productoras que durante el año 2013 han participado en la producción de películas únicamente cuatro de ellas han producido 5 o más películas, 37 empresas entre dos y cuatro películas y el mayor número de ellas 226, una sola película. Dichos volúmenes de producción discontinuos y poco diversificados (una tendencia del sector español) "dificulta la realización de economías de escala para optimizar recursos y resultados” (ICAA, 2014:18). De 
hecho, se considera que hasta que no se consigan unos volúmenes de producción sistemáticos "no se creará una industria cinematográfica capaz de rentabilizar inversiones, diversificar riesgos y consolidar su permanencia” (ICAA, 2014:18).

La producción de películas y ficción televisiva no es únicamente un importante sector económico en cuanto a su capacidad para crear empleo y riqueza sino que también como indica Gillian Doyle en su artículo "Economía audiovisual: los mercados audiovisuales en la Unión Europea" es necesario valorar su potencial para ayudar al crecimiento del PIB ya que en "el contexto de la estrategia de Lisboa de 2010, fue visto como un importante catalizador para un mayor crecimiento económico" (Doyle, 2012:16). Además, como uno de los componentes de la denominada economía digital "puede contribuir a la estrategia de revitalización económica y de crecimiento "Europa 2020”'” (Doyle, 2012:16). Por otra parte, a la hora de evaluar el peso del sector es preciso también incluir el relevante papel que el sector desempeña "en el mantenimiento de las identidades y la promoción de la tolerancia y la cohesión social” (Doyle, 2012:16). Por lo tanto, como señala Enrique Bustamante en su publicación "España: La cultura en tiempos de crisis. Fuentes financieras y políticas públicas", la producción audiovisual, como toda producción de un bien cultural, asume en las sociedades contemporáneas un doble papel: "la cultura como derecho (de acceso, de diversidad) en tanto base fundamental de la democracia y la cultura como recurso económico directo, como sector vital para una sociedad del conocimiento" (Bustamante, 2013:5).

El modelo de financiación por medio del crowfundig (CF), puede ser una de las posibles soluciones para resolver los problemas de financiación y de esta forma poder incrementar su oferta.

\section{EL CROWFUNDING}

La Asociación Española de Crowfunding (SCF), lo define como "un sistema de cooperación que permite a cualquier persona creadora de proyectos, reunir una suma de dinero entre muchas personas para apoyar una determinada iniciativa empresarial, cultural, social o deportiva” (SCF,2014:4). Esta definición está incluida en su publicación "Informe de la encuesta de la Asociación Española de Crowdfunding sobre plataformas española de crowfunding", donde se recogen una serie de datos contrastados muy interesantes sobre la situación del CF en España durante el año 2013, el año en que se realizó la encuesta e información que se utilizará ampliamente a lo largo de este artículo, aunque se contrastará con la proporcionada por otras fuentes. Por otra parte, la SCF es la asociación de profesionales y empresas del sector del CF del Estado Español

Por su parte, Thomas Lambert y Armin Schwienbacher en su publicación “An Empirical Analysis of Crowdfunding”, que es un Documento de Trabajo de la Louvain School of Management (Université Catholique de Louvain) mencionan que el concepto de CF tiene su raíz en un concepto más amplio: en el concepto de crowdsourcing, que lo definen como recurrir a la "multitud" para obtener información, 
ideas y soluciones para el desarrollo de las actividades empresariales (Lambert y Schwienbacher, 2010:2)

Lambert y Schwienbacher comentan que en el caso del CF el objetivo es poder recaudar financiación para una inversión. Pero en lugar de recurrir a un pequeño grupo de inversores se obtienen los fondos necesarios de una audiencia muy elevada: la multitud, donde cada persona proporcione una cantidad reducida. Por lo tanto, el elemento determinante del CF es recaudar fondos de una multitud que proporciona una ayuda financiera para el desarrollo de un proyecto. Dicha multitud a veces también participa en las decisiones estratégicas del proyecto e incluso tiene derecho a voto. Se recurre a ella por medio del uso de Internet, básicamente gracias a las redes sociales. Además han surgido distintas plataformas que actúan de intermediarias entre los promotores y la multitud.

En su artículo Lambert y Schwienbacher especifican que aunque se utiliza de forma mayoritaria en la industria del entretenimiento (en especial en la música y las películas), también hay iniciativas en otras industrias, entre las que mencionan: periodismo, software, libros, alimentación y moda (Lambert y Schwienbacher, 2010:2). En la mayoría de los proyecto de CF que analizan en su artículo, las personas que participan aportando financiación realizan lo que estos autores denominan como unas inversiones pasivas en las que no participan en el proceso de toma de decisiones del proyecto. Son inversiones con una promesa por parte del promotor de una compensación, que en la mayor parte de las ocasiones, se trata de recibir un servicio o producto de la actividad financiada (Lambert y Schwienbacher, 2010:3).

En el informe elaborado por la SCF se comenta que se puede hablar de cuatro tipos distintos de CF en función de la naturaleza del intercambio:

Donación: “enfocado a proyectos solidarios humanitarios, los donantes aportan un importe a un proyecto en concepto de donación, sin ninguna contrapartida" (SCF, 2014:4). El 21,1\% de las plataformas de CF que han respondido a la encuesta de SCF se dedican a las donaciones. La aportación media de las personas que han participado en sus campañas es de 34,19 euros.

Recompensa: "enfocado al desarrollo de productos creativos, el mecenas hace una aportación a cambio de una compensación no dineraria. Se considera una preventa”. (SCF, 2014:4). Es la modalidad de CF más extendido, cerca de la mitad, el 41,4\% de las plataformas incluidas en el informe. También es el tipo preponderante a nivel mundial. La aportación media de los mecenas es de 35,82 euros.

Inversión: "el inversor o micro-inversor recibe una acción de una empresa, una participación de la misma, o compromisos sobre beneficios de la misma, como contraprestación a su aportación”. (SCF, 2014:4). Su peso relativo en España, es del 20,7\%. La aportación del inversor medio es muy elevada: 4.853,33 euros 
Préstamo: "el prestamista recibe su préstamo junto con un tipo de interés previamente acordado como contraprestación a su aportación.” (SCF, 2014:4). Se puede centrar tanto en particulares como en empresas y suponen el menor porcentaje:13,8\% de las plataformas españolas. Su inversor medio realiza, al igual que en la modalidad anterior de CF, una elevada aportación: 2.025,5 euros.

Cuando la exposición se centra en la comparación de la cantidad demandada por los distintos proyectos de las diferentes modalidades de CF, también se puede apreciar cómo se producen unas elevadas dispersiones en la cantidad media que se ha solicitado ya que "de media, los proyectos de recompensa y donación que tienen éxito, solicitan alrededor de 3.300 euros y fracasan los que sobrepasan los 5.500 euros. Para préstamos e inversiones las cifras son superiores; tienen éxito los proyectos que solicitan alrededor de los 30.200 euros y fracasan los que piden más de 200.000 euros” (SCF, 2014:4).

Las plataformas realizan una labor de intermediario entre la multitud financiadora y los emprendedores y por ello cobran una comisión que es un porcentaje del importe recaudado por los proyectos que han tenido éxito. En España, y según la SCF el promedio de comisiones oscila entre el 3,5\% en los proyectos de donaciones, del $5 \%$ al 6\% en los casos de recompensa y préstamos y se eleva hasta el 6,4\% en los de inversión. Por otra parte, se estima por parte de la SCF una recaudación por medio de CF de 19 millones de euros en todo el Estado Español durante el año 2013 (SCF, 2014:4).

Por su parte, en el ámbito europeo se considera que el volumen de transacciones realizadas gracias a la financiación alternativa por medio de plataformas online se multiplicó por seis entre los años 2012 y 2014, desde los 500 millones de euros hasta los 3.000 y se prevé que alcanzará los 7.000 en el año 2015, según la información que proporciona el estudio "Moving Mainsttream. The European Alternative Finance Benchmarking Repor" realizado por los investigadores Robert Wardrop, Bryan Zhang, Raghavendra Rau y Mia Gray del Cambridge Centre for Alternative Finance. Si se desagrega esta cifra entre los 27 países europeos estudiados, se puede apreciar que el primer lugar lo ocupan las plataformas británicas que han conseguido recaudar 3.560 millones de euros.

En la siguientes posiciones, aunque con volúmenes mucho más reducidos (expresados en millones de euros) se sitúan Francia con 253, Alemania: 236, Suecia: 207, Holanda: 155 y España con 101 (Wardrop et al., 2015:15). Los autores ponen como ejemplo de los desiguales grados de desarrollo de la financiación alternativa en los distintos países europeos el hecho de que la suma del volumen de financiación de estos cinco mencionados países: 952 millones de euros, es 6,7 veces mayor que la suma correspondiente a las cifras de los 21 países restantes. En relación a las cifras logradas por los distintos tipos de modelos de financiación alternativos (excluyendo en el análisis al Reino Unido) el segmento que tiene un mayor volumen son los préstamos de consumo con una cifra de 274,62 millones de euros en el año 2014, seguido 
por el crowdfunding recompensa con 120,33, en tercera posición los préstamos a empresas con 93,1 y a continuación el CF inversión con 82, 56 millones de euros (Wardrop et all, 2015:9).

Aunque el CF puede generar al promotor unos costes de transacción, que en algunos casos pueden llegar a ser elevados, en comparación con los que tienen otros sistemas de financiación, Lambert y Schwienbacher consideran que también tiene una serie de ventajas. Para ellos la mayor ventaja de este sistema de financiación es la atención que dicho promotor puede atraer hacia su proyecto. Este hecho es un activo vital especialmente en el caso de los artistas que de esta manera pueden presentar las cualidades de sus productos a la multitud, que a su vez es su clientela potencial (Lambert y Schwienbacher, 2010:12). El CF facilita que los artistas puedan validar y testar sus productos ante su audiencia potencial y poder observar de esta forma cómo reacciona ante ellos, lo que proporciona una relevante información sobre el mercado potencial del producto ofertado.

Por su parte, Daniel Oliver, que es el Presidente de la SCF y miembro del Consejo de la European Equity Crowdfunding Associatión, en su colaboración incluida en la investigación de la Cambridge Centre for Alternative Finance, anteriormente mencionada, resalta la importancia que está adquiriendo la economía colaborativa en la economía española. También indica el relevante papel que a su juicio está desempeñando el CF ayudando a los artistas españoles y reemplazando el papel de la financiación pública, actualmente muy reducida (Wardrop et al.,2015: 32). En relación a ello, Antoni Roig comenta en su artículo "La participación como bien de consumo: una aproximación conceptual a las formas de implicación de los usuarios en proyectos audiovisuales colaborativos” el hecho de que la participación de los usuarios es una de las principales características de la actual producción cultural.

Dentro de los proyectos audiovisuales basados en la participación, Roig distingue entre dos tendencias, una que facilita que los usuarios puedan compartir y subir vídeos por medio de plataformas. Y una segunda tendencia "que vincula producción y participación: la emergencia de proyectos concebidos a partir de la implicación individual o colectiva de su público objetivo en diferentes fases del mismo" (Roig, 2010:104). En este artículo se distingue entre dos categorías de proyectos audiovisuales basados en la participación en función del grado de implicación que se permita a los usuarios. Hay una primera categoría "en la que se propone una descentralización controlada de una pequeña parte de la actividad creativa, que en términos generales se traduce en unas reglas de juego predefinidas” (Roig, 2010:106).

Y una segunda categoría con proyectos "impulsados con la intención de implicar de forma efectiva a su público en distintas fases del proyecto... Se trata por tanto de productos donde la implicación de los públicos tiene una influencia tangible en el proceso y en el producto" (Roig, 2010:106). Se menciona dentro de esta categoría al proyecto español de El cosmonauta como ejemplo de un modelo que combina tanto el CF con inversión privada y licencias creative commons, cuya "comunidad se construye a partir de una suscripción simbólica, que convierte a los usuarios en coproducto- 
res, actuando básicamente como prescriptores del proyecto, aunque tomando parte en diferentes convocatorias para la toma de decisiones sobre el film” (Roig, 2010:107).

A su vez Michel Barnier resalta el papel que el CF puede tener en la financiación de proyectos de las industrias culturales. En su intervención en el ciclo de conferencias denominado Crowfunding: untapping its potential; reducing the risks” organizado por la Comisión Europea en junio de 2013, afirmó que el CF es un catalizador de innovación además de resaltar entre sus efectos secundarios positivos la posibilidad que presenta de poder testar el producto y poder convertir a los clientes potenciales del producto en inversores del proyecto. Michel Barnier considera que el CF es un instrumento de financiación muy adecuado para promover el nuevo modelo de crecimiento propuesto por la estrategia comunitaria 2020.

El primer proyecto cinematográfico financiado por CF fue la película francesa Lugar para ayer, que en tres semanas consiguió recaudar 60.000 euros en el año 2004. Se establecieron una serie de recompensas como poder asistir al rodaje, dvd, la posibilidad de asistir a la fecha del estreno de la película, entre otras. En el año 2013 Inocente obtuvo un premio Oscar al mejor cortometraje documental. Fue realizado por Andrea Fine y Sean Fine y financiado por los internautas a través de la plataforma Kickstarter, por medio de 300 donantes reunieron alrededor de 50.000 dólares.

El CF no es únicamente un modelo de financiación utilizado por los directores emergentes, dos buenos ejemplos de ello son: David Fincher, productor del film animado The Goon que recaudó 450.000 vía Kickstarter para adaptar el cómic al cine. Y Charlie Kaufman, que consiguió 406.237 dólares -el doble de lo que necesitabapor esta vía para la adaptación animada de Anomalisa.

En relación al cine español, el primer proyecto financiado fue Los amores difíciles de Lucina Gil, un corto del 2013, que estuvo nominado al Goya. También se financió con esta modalidad el largometraje de ciencia ficción El cosmonauta, con un presupuesto de 400.000 euros, de los que 250.000 los aportaron 4.000 inversores anónimos contactados por internet. Y O apostolo, el primer largometraje de animación stopmotion español. También tres obras presentes en los Goya de 2014 obtuvieron financiación vía CF: El paraguas de colores, Las maestras de la República y Stokholm. Y en el año 2015 Juan y la nube obtuvo el Goya al Mejor corto de animación

Una prueba del grado de implantación que está empezando a conseguir el CF en la financiación de esta industria cultural en España es que de los 2.080 proyectos que consiguieron financiación en la plataforma Verkami, que es una plataforma española especializada en proyectos culturales, 769 son proyectos cinematográficos 


\section{PEQUEÑOS MECENAS PARA GRANDES PROYECTOS: JUAN Y LA NUBE, UN EJEMPLO DE $\mathrm{CF}^{1}$}

El 7 de febrero de 2015, el cortometraje Juan y la nube, un proyecto de Zampanò Producciones, se alzó con el Goya al Mejor corto de animación. Justo un año antes, sus creadores estaban en plena campaña de CF para conseguir terminar este trabajo. A continuación, se explicará el proceso llevado a cabo para poner en marcha esta forma de financiación colectiva, que permitió a sus productores finalizar con éxito el cortometraje, después de más de dos años de trabajo.

\subsection{Primeros pasos: ¿cómo empezar un CF?}

Conseguir que alguien - un amigo o familiar - ponga dinero para que puedas producir un corto es algo relativamente fácil, sobre todo si uno tiene muchos amigos en Facebook o muchos seguidores en Twitter. Lo difícil es conseguir que gente que no te conoce, que no ha visto tu trabajo, decida darte dinero para que tu proyecto audiovisual vea la luz. Ese es el reto de cualquier CF: en cuarenta días es necesario convencer a mucha gente de que merece la pena apostar, aunque sea una cantidad pequeña, por un proyecto y una productora que tal vez no conozcan. Este fue el reto que tuvieron que superar los productores de Juan y la nube: conseguir el objetivo económico de diez mil euros en cuarenta días.

Para ello, es fundamental hacer una buena presentación del proyecto: ¿Qué es Juan y la nube?:

"Juan y la nube es un proyecto cinematográfico de animación stop-motion. Un cortometraje que adapta un cuento infantil con un atrevido estilo visual donde los muñecos animados se integran con fondos reales. Una historia en defensa del medio ambiente donde el poder de la imaginación se enfrenta a la insensibilidad de los adultos" (extraído de la página del CF de Juan y la nube).

Estas primeras cincuenta palabras son fundamentales ya que son las primeras que leen los posibles financiadores. Tiene que ser un mensaje claro, conciso, pero a la vez atractivo y lo suficientemente interesante para que los usuarios pinchen en el enlace para saber más.

${ }^{1}$ Carlota Coronado, coautora de este artículo participó en el CF como Community Manager de toda la campaña de CF en redes. Además coordinó toda la campaña (realización de vídeos, envío de notas de prensa, mailing...). Por otra parte, es co-productora del corto junto a Giovanni Maccelli y directora de producción del corto. 
Para quienes deciden hacer click, es necesario ofrecerles una información más detallada, que no sólo describa el proyecto, sino que presente a sus impulsores. Se debe explicar el proyecto, el estado de desarrollo en el que está - preproducción, rodaje o postproducción - y los tiempos de elaboración una vez acabado el CF. Es fundamental dejar muy claro estos aspectos para generar confianza en el futuro inversor: en el caso de Juan y la nube se indicaron los procesos de producción que se realizarían - retoque de imagen, composición de capas, procesos de render, montaje final y etalonaje- y se dieron fechas de entrega de copia final y de estreno (julio 2015). La indicación de un calendario de trabajo es fundamental para generar confianza, ya que hace más tangible la recompensa final - asistir al estreno o tener el dvd, por ejemplo. Otro de los aspectos que se subrayó a la hora de presentar Juan y la nube fue la participación de instituciones como el Ministerio de Cultura y la Comunidad de Madrid en la financiación del cortometraje. Mostrar a posibles inversores que el corto está respaldado económicamente por estos dos entes no sólo genera mayor confianza y confiere más seriedad al proyecto, sino también más calidad. Son pocas las obras audiovisuales que cada año reciben ayudas estatales y autonómicas, y deben pasar un proceso de evaluación de carácter competitivo entre centenares de solicitantes.

Pero lo que más confianza produce en los futuros mecenas es conocer bien a las personas que están detrás del proyecto. Cuando pago algo a través de Internet, necesito saber si puedo fiarme. Es por ello que en todo proyecto de CF resulta imprescindible hacer una buena presentación de los productores o creadores de la obra. En el caso de Juan y la nube, la productora estaba avalada por los más de doscientos premios recibidos por cortos anteriores, lo que la había convertido en una de las productoras clásicas de cortometrajes dentro del audiovisual madrileño. Aun así, para quienes no conocieran ningún trabajo producido por Zampanò, se ofrecía información adicional con enlaces a la web de la productora, a su canal de Youtube - en el que se podían visionar los cortos -, así como a las redes sociales asociadas al proyecto y sus creadores. Otras de las claves fundamentales para que un proyecto de CF sea atractivo es que tenga suficiente material audiovisual para que el público - posible inversor pueda ver cómo será el resultado final. Resulta mucho más complicado conseguir apoyos para un proyecto que todavía está sólo en papel que para otro - como es el caso de Juan y la nube - que está ya rodado. Esta fue una de las grandes ventajas: poder mostrar fotografías del corto, imágenes del making of o partes con la postproducción ya finalizada para que el proyecto fuera más atractivo.

El director y co-productor de Juan y la nube llevó a cabo la edición de un tráiler en el que se mostraba de un modo sencillo y claro en qué consistía el proyecto y para qué era necesario el dinero del CF (ver imagen 1). Este tipo de vídeos, de una duración de entre uno y tres minutos, son fundamentales en una campaña de micromecenazgo por diferentes razones. En primer lugar, porque las piezas audiovisuales resultan más atractivas de cara a su difusión a través de redes sociales, la base de cualquier campaña de financiación colectiva. En segundo lugar, colgar un tráiler en Youtube permite a los impulsores de la campaña, contabilizar las visitas diariamente, lo que ayuda a 
saber si la difusión está teniendo buena respuesta o es necesario buscar nuevas estrategias comunicativas.

Pero antes de poner en marcha la campaña y que empiece la cuenta atrás para llegar al objetivo económico establecido, es necesario elegir las diferentes recompensas a las que podrá optar quien quiera participar económicamente. Es importante ofrecer un amplio abanico de recompensas, para todos los bolsillos: desde 5 hasta $1.000 €$. Además de las tradicionales, como incluir el nombre del mecenas en la página web de la productora, en los títulos de crédito o la entrega de dvd y demás material gráfico - postales, imanes, pressbook, etc. -, es importante pensar en recompensas atractivas que no sólo sean materiales. Así, por ejemplo, los mecenas de Juan y la nube podían elegir hacer un curso intensivo de stop-motion impartido por el director del corto. De esta manera, uno se convierte en micro-productor dando dinero para un proyecto de cortometraje, pero a cambio recibe un taller intensivo de seis horas. Con esto, los apoyos económicos se extendían más allá de los círculos de amigos y familiares: cualquiera interesado en la animación y en recibir un curso de esta materia podía convertirse en micro-productor. Con este tipo de recompensas, no sólo se llega a un público potencial más amplio, sino que hace que el CF no sea una mera donación, sino una contraprestación.

La elección de recompensas atractivas resulta, por tanto, muy útil de cara a la campaña de difusión del CF. A lo largo de los cuarenta días del proceso, se van creando contenidos al darse a conocer nuevas recompensas: para el 14 de febrero, los productores de Juan y la nube, difundieron una nueva recompensa -"Regala un mensaje eterno en San Valentín". Consistía en incluir un mensaje de amor en un graffiti en la calle, que se incluiría en postproducción en alguna secuencia del corto (ver imagen 2).

El último requisito antes de poner en marcha el CF es establecer el objetivo de financiación, por lo que es necesario llevar a cabo un presupuesto de los futuros gastos del proyecto, que se sufragarán a través de las aportaciones de los mecenas. La mayor parte de las producciones audiovisuales que han conseguido finalizar con éxito su CF han conseguido entre 2.500 y 5.000 euros. Por encima de esta cifra, sobre todo si se trata de cortometrajes, resulta mucho más complicado llevarlo a buen fin. Para Juan y la nube, después de elaborar un presupuesto, se estableció la cifra que se pretendía alcanzar en $10.000 €$, ya que era la cantidad necesaria para poder terminar todos los procesos de postproducción del corto. Este objetivo de financiación supone conseguir doscientos cincuenta euros al día. Para conseguirlo era necesario poner en marcha una buena campaña de marketing, pero prácticamente a coste cero. 


\subsection{Cuarenta días de infarto: ¿cómo hacer una campaña de CF y no morir en el intento?}

Cuando el promotor de un CF establece su objetivo económico - diez mil euros, por ejemplo - sabe que tan sólo tiene cuarenta días para conseguirlos ${ }^{2}$. Gran parte del éxito de un proyecto reside en su buena difusión. Por ello, antes de lanzar el proyecto a la campaña de cuarenta días para la captación de financiadores, es muy importante contar con una planificación previa de difusión del mismo y realizar un calendario estructurado de acciones relacionadas.

Para Juan y la nube se elaboró un plan semanal de difusión: desde la presentación del proyecto, al lanzamiento de nuevos vídeos cada semana, nuevas recompensas o contenidos relacionados con el proyecto publicados en medios. El objetivo era crear continuamente contenidos nuevos que sirvieran de recordatorio al público potencial, sin resultar demasiado insistentes: el bombardeo informativo puede resultar contraproducente en una campaña de estas características.

Para articular y llevar a cabo una campaña eficaz era necesario analizar a priori qué canales se iban a utilizar: mailing, redes sociales (Facebook, Twitter, blogs), prensa escrita o digital, presentaciones informales, whatsapp, etc. Está claro que sin redes sociales no puede haber $\mathrm{CF}$, porque son los únicos canales que permiten una difusión amplia a un coste muy reducido. Por ello, antes de nada, es imprescindible crear una página de Facebook del proyecto - Juan y la nube, en este caso - e invitar a todos los contactos a decir "Me gusta". De esta manera, cada post, foto o vídeo publicados en el muro de esta página llegarían a los seguidores de la página.

El pilar de la campaña fue, por lo tanto, Facebook, sobre todo como base para la difusión de contenido para compartir. Pero también se hizo uso de otras redes sociales como Twitter o Google+. A éstas se unió la fórmula tradicional de la difusión en medios como prensa digital o blogs. Para ello se elaboraron notas de prensa que se enviaron a revistas o publicaciones especializadas en cine o animación. Las noticias sobre el CF de Juan y la nube que se publicaban en prensa digital alimentaban de contenido la página de Facebook y la cuenta de Twitter.

Una clave para que la campaña de comunicación funcione es elegir bien el público objetivo y saber cuáles son los mejores canales para llegar a él. En el caso de Juan y la nube, la técnica cinematográfica marcaba ya un target muy claro: amantes de la

${ }^{2}$ El tiempo de duración de un CF está establecido desde la plataforma que lo gestiona. Por lo general, suelen durar todos cuarenta días, pero hay excepciones: plataformas que ofrecen el doble de tiempo estableciendo dos objetivos económicos - uno mínimo para los primeros cuarenta días y si se llega a este objetivo, se activan otros cuarenta para alcanzar la segunda cifra -; otras que si se llega al 90\% del objetivo antes del último día, conceden cuatro días más para que el proyecto finalice con éxito, etc. 
animación, profesionales del stop-motion y de la animación en general. Así pues, se difundieron los mensajes y vídeos de la campaña a través de grupos de Facebook como "Stopmotia. Comunidad del Stop-motion", "Spanish animators", "Stop-motion Argentina" o "SMIC (Stop Motion International Community)", entre otros. Cado uno de estos grupos tiene más de dos mil miembros, por lo que la publicación en el muro de estas comunidades permite una gran difusión en términos cuantitativos además de la internacionalización de la campaña ${ }^{3}$.

Además de buscar posibles financiadores entre los aficionados al mundo de la animación, se tuvieron en cuenta otras características del proyecto que permitían llegar a nuevos públicos: se trataba de un corto de animación infantil con temática relacionada con la defensa del medio ambiente. Esto abrió otros campos para la difusión: blogs, publicaciones o grupos de Facebook dedicados a cine o actividades para niños, por un lado; y por otro, organizaciones o asociaciones ecologistas que pudieran, bien contribuir económicamente, bien ayudar a la difusión del proyecto.

Otro de los métodos de difusión fue el mailing. Se llevaron a cabo tres oleadas de mails: la primera para la presentación del proyecto; la segunda, hacia el ecuador de la campaña, para informar sobre el desarrollo de la campaña y hacer un recordatorio; y la tercera y última, unos días antes del cierre, para anunciar la cuenta atrás y animar a los rezagados que lo han dejado para el final. De esta manera, en cuarenta días, los posibles financiadores reciben tan sólo tres mails y no son bombardeados. El abuso del mailing puede tener un efecto negativo y llevar el mail directamente a la papelera de reciclaje o al anti-spam. Para Juan y la nube, además de mails masivos a todos los contactos, se hizo un mailing más selectivo: se buscaron los correos de miembros de asociaciones de cortometrajistas - como la Coordinadora del Cortometraje - o de personas o productoras relacionadas con el mundo de la animación.

A través de estos canales, se configuró una red de contactos que a su vez difundían también - reenviando mails o compartiendo en redes sociales - los contenidos de la campaña. La expansión de ésta a cada vez más público se hacía palpable numéricamente a través de las visitas diarias de los vídeos colgados y de la cifra de personas alcanzadas que Facebook ofrece para cada publicación en la página de Juan y la nube. Esta herramienta permitía ver qué tipo de contenido tenía más éxito: las fotografías del rodaje, por ejemplo, tenían de media un alcance de entre ochocientas y mil personas, mientras que los textos o incluso algunos vídeos no llegaban a más de trescientas. Gracias a estos datos, se modificaron las acciones previstas, potenciando la publicación del contenido más demandado (por ejemplo, la serie de fotos de antes - con chroma- y después de la postproducción, que produjo mucha curiosidad).

\footnotetext{
${ }^{3}$ La difusión de la campaña fuera de las fronteras españolas permitió conseguir mecenas de otros países como Alemania, Italia o Argentina.
} 
Se llevó a cabo, por tanto, un seguimiento diario y continuado de todas las acciones comunicativas y del desarrollo del CF, desde su lanzamiento hasta el final de la campaña de captación de financiadores de los 40 días. Esta limitación temporal es un elemento esencial para conseguir el objetivo económico, puesto que si no hay una fecha de cierre, la gente suele posponer su aportación y la campaña podría tardar meses en llegar a su final. Así, por lo general, la mayor parte de los CF que terminan con éxito suelen conseguir el 30\% del objetivo económico en la primera semana. Seguidamente pasan por un desierto en las semanas centrales, y es en los últimos días cuando se aceleran las aportaciones. La campaña de comunicación se adecua también a estas fases y utiliza el recurso de la cuenta atrás - "últimas 72 horas para participar", por ejemplo -, para animar a los inversores a hacer sus contribuciones.

Para hacer realidad Juan y la nube fue necesaria la aportación de 175 mecenas. Personas de diferentes edades, clases sociales y países, unidas por un objetivo común: ayudar a la producción de un proyecto cinematográfico como Juan y la nube. Dejaban de ser meros espectadores para convertirse en micro-productores. Así, proyectos grandes y pequeños ven la luz gracias a un granito de arena, porque los granitos de arena, hacen montañas.

\section{ANÁLISIS DE LAS PRINCIPALES CARACTERÍSTICAS DE LOS MECENAS}

En primer lugar se van a exponer los principales motivos que llevan a los mecenas a financiar proyectos por medio de CF y para ello se utilizará la información proporcionada por el informe “¿Por qué la gente financia proyectos vía crowfunding? Investigación realizada entre la Comunidad de Mecenas de Lánzanos” llevada a cabo por Luis Miguel Barral y Pepa Barral. Este trabajo se basa en una encuesta realizada en marzo del 2014 entre la comunidad de usuarios registrados en Lánzanos que es una plataforma española de CF más importantes. Se analiza la opinión de una muestra de 3.153 usuarios de Lánzanos que en el periodo de los tres últimos años han contribuido como mecenas en algún proyecto.

Los principales motivos (clasificados por orden de importancia) aducidos por los mecenas para financiar proyectos vía CF son: 1) Contribuir a crear lo que me gusta 2) Apoyo directo a los creadores, sin intermediarios 3) Contribuir a crear cosas innovadoras 4) Apoyar al impulso emprendedor. En dicho informe se indica que "lo que hace "sentir bien al mecenas" es sinónimo del beneficio que obtiene al sumarse a esta corriente social. Los datos no indican que el beneficio del crowfunding para el mecenas es básicamente una historia de cambio hacia algo nuevo y valioso" (Barral y Barral, 2014:83)

Un aspecto muy relevante es que el mecenas decide la cuantía que aporta al proyecto teniendo en cuenta lo que recibe a cambio, de manera que "las aportaciones sin recompensa cosechan cuantías individuales inferiores a las aportaciones donde hay 
recompensas de carácter simbólico; y estas cosechan cuantías inferiores a las que se basan en la pre-compra de un bien o servicio que el promotor se compromete a crear y entregar al mecenas bajo unas condiciones” (Barral y Barral, 2014: 56). De hecho se especifica que "el formato del intercambio tiene una influencia decisiva" (Barral y Barral, 2014: 54) en la cantidad aportada por el mecenas. Es también muy relevante el interés que un determinado proyecto suscita , de hecho es más determinante para la cuantía el interés del proyecto que las vinculaciones familiares o de amistad con el promotor, debido a ello "cuando un mecenas se interesa por un proyecto (y no sólo por el vínculo con el promotor) la cuantía tiende a ser mayor porque está contribuyendo a un intercambio de algo que tiene valor "per se" y, en consecuencia, aporta la cuantía que se considera conveniente, "sin compromisos" ajenos al valor que lo financia” (Barral y Barral, 2014:55).

Tabla 1. Perfil de la audiencia del cine y de los mecenas de Juan y la nube.

\begin{tabular}{|c|c|c|c|}
\hline & & Cine & Juan y la nube \\
\hline SEXO & Hombre & 49.6 & 42.8 \\
\hline & Mujer & 50.4 & 57.2 \\
\hline EDAD & $<20$ & 12.1 & 0.0 \\
\hline & $20-24$ & 9.9 & 1.7 \\
\hline & $25-34$ & 25.0 & 4.6 \\
\hline & $35-44$ & 21.6 & 56.0 \\
\hline & $45-54$ & 13.3 & 11.4 \\
\hline & $55-64$ & 9.0 & 14.3 \\
\hline & 65 ó más & 8.9 & 12.0 \\
\hline
\end{tabular}

Fuente: Elaboración propia y AIMC "Estudio General de Medios ( $3^{\mathrm{a}}$ ola) Febrero a Noviembre de 2013 Resumen General”.

A continuación se realiza en esta exposición un análisis sociodemográfico de los mecenas que han financiado el proyecto de Juan y la nube en primer lugar el análisis se centra en la comparación de sus principales características sociodemográficas con el perfil de la audiencia de cine y para ello se utilizará la información que proporciona la Asociación para la Investigación de Medios de Comunicación (AIMC) en su informe "Estudio General de Medios ( $3^{\mathrm{a}}$ ola) Febrero a Noviembre de 2013 Resumen General”. La audiencia se mide como la asistencia al cine la semana anterior a la realización de la encuesta por la AIMC. Se aprecia de forma inmediata que existen elevadas disparidades entre los valores de las características de ambos colectivos (tabla 1). Con respecto a la variable sexo, las mujeres representan un mayor peso porcentual en el colectivo de los mecenas que en la audiencia del cine y en consecuencia en el caso de los hombres ocurre la situación contraria. Mayores divergencias se presentan en relación a la variable edad. Mientras que la audiencia del cine se 
concentra entre las personas que tienen de 25 a 44 años y con un grado algo menor entre 45 y 54. Por el contrario, en los mecenas aunque es destacable el elevado peso de los que tienen de 35 a 44 años, los siguientes colectivos con mayor representación son los que tienen una mayor edad, más de 55 años, cuya audiencia al cine, en cambio, es la más reducida de todos los estratos de edad. También es reseñable el pequeño peso que tiene entre los mecenas el colectivo entre 25 y 34 años, que por el contrario es el tramo de edad que goza de un peso superior en cuanto audiencia de cine.

Tabla 2. Perfil de los mecenas de la plataforma Lánzanos y de Juan y la nube.

\begin{tabular}{|l|l|c|c|}
\hline & & Lánzanos & Juan y la nube \\
\hline SEXO & Hombre & 73.3 & 42.8 \\
\hline & Mujer & 26.7 & 57.2 \\
\hline EDAD & $18-29$ & 19.9 & 4.0 \\
\hline & $30-39$ & 47.0 & 38.3 \\
\hline & $40-49$ & 21.7 & 30.3 \\
\hline NIVEL & $50 \mathrm{y}+$ & 11.4 & 27.4 \\
\hline ESTUDIOS & Estudiantes & 4.0 & 2.3 \\
\hline FINALIZADOS & No universit & 27.7 & 16.0 \\
\hline
\end{tabular}

Fuente: Elaboración propia y Luis Miguel Barral y Pepa Barral “¿Por qué la gente financia proyectos vía crowfunding? Investigación realizada entre la Comunidad de Mecenas de Lánzanos”.

En cambio, se produce un mayor grado de coincidencia del perfil de los mecenas de Juan y la nube con el que tienen los usuarios de la red social Facebook (que se utilizó de forma intensiva en el proceso de CF del proyecto) según la información que proporciona el informe "IV Estudio anual de Redes sociales" de la Interactive Advertising Bureau (IAB). En ambos casos es mayor el peso en términos porcentuales de las mujeres, aunque es algo superior en el caso de los usuarios de Facebook (62\%) que en el de los mecenas (57\%). En relación a la edad, el tramo más relevante en los perfiles son las personas con una edad comprendida entre los 30 y 39 años (IAB, 2013:35). Por el contrario el tramo de los individuos entre 18 y 29 años, muy relevante en Facebook (con el 34\%), sólo tiene un peso del 4\% en el colectivo de los mecenas.

Por último, cuando se compara el perfil de los mecenas de Juan y la nube con el perfil medio de la muestra de mecenas de la plataforma Lánzanos (tabla 2), en primer lugar resalta el elevado peso porcentual que representan los hombres con 73,3\% en el colectivo de mecenas de la plataforma, en cambio, entre los mecenas de Juan y la nube es algo superior el peso de las mujeres $(57,2 \%)$ que el que tienen los hombres. En relación a la edad, el tramo más relevante en ambos casos son los individuos con una edad comprendida entre los 30 y 39 años, seguido por los que tienen entre 40 y 
49. Entre el colectivo de mecenas de Lánzanos el siguiente tramo en importancia, son las personas más jóvenes de 18 a 29 años, colectivo que tiene un peso muy reducido entre los mecenas de Juan y la nube. Pero ocurre lo contrario en el tramo de las personas de más edad $(50 \mathrm{y}+$ ). Por último, en relación a la variable nivel de estudios finalizados por los mecenas se produce un mayor grado de igualdad en la comparación del perfil de ambos colectivos (tabla 2) aunque es superior el nivel medio de formación entre los mecenas de Juan y la nube, con un peso porcentual del 81,7\% entre las persona que tienen formación universitaria, por el 68,3\% entre los mecenas de Lánzanos.

\section{CONCLUSIONES}

La crisis económica ha provocado que el audiovisual español atraviese una dura situación. Se considera que el cine y la ficción televisa están en un estado que ha sido calificado como de crisis estructural. Y el sector de la producción tiene graves problemas de solvencia financiera.

La producción de películas y ficción televisa no sólo tiene importancia por su capacidad para crear empleo y riqueza, sino que también tiene un relevante papel potenciando un mayor crecimiento económico, siendo uno de los componentes básicos de la economía digital como se señala en las estrategias Comunitarias de Lisboa 2010 y Europa 2020. Así mismo desempeña una importante misión manteniendo las identidades y promocionando la cohesión social y tolerancia. La producción audiovisual, como toda producción de un bien cultural, asume un doble papel: como derecho (de acceso, de diversidad) y como recurso económico.

El modelo de financiación por medio del CF puede ser una de las posibles soluciones para resolver los problemas de financiación del audiovisual español y de esta forma poder incrementar su oferta.

Aunque el CF se ha utilizado primordialmente en la industria del entretenimiento, especialmente financiando películas y música, existen muchas iniciativas en otras industrias. El CF, no solo permite obtener financiación para el proyecto, sino que también facilita que se pueda testear el producto ante su audiencia potencial, lo que proporciona una relevante información sobre el mercado potencial del producto ofertado.

El CF se está implantando paulatinamente en la producción audiovisual, tanto a nivel de España, como en otros países. Además, varios proyectos financiados con este modelo de financiación han conseguido diferentes premios. Una prueba del grado de implantación que está empezando a conseguir el CF en la financiación de esta industria cultural en España, es que de los 2.080 proyectos que consiguieron financiación en la plataforma Verkami, una plataforma española especializada en proyectos culturales, son proyectos cinematográficos 769 . 
Para conseguir el éxito en una campaña de CF es fundamental hacer una buena presentación del proyecto, así como de los productores o creadores de la obra. Con una información muy detallada para generar confianza en el futuro inversor. La participación de instituciones de prestigio en el proyecto también contribuye a generar una mayor confianza.

Para conseguir que el proyecto resulte atractivo hay que mostrar suficiente material audiovisual para que el público -posible inversor- pueda ver de un modo sencillo y claro en qué consistía el proyecto y para qué era necesario el dinero del CF. Además, las piezas audiovisuales resultan más atractivas de cara a su difusión a través de redes sociales, la base de cualquier campaña de financiación colectiva.

Es necesario elegir las diferentes recompensas a las que podrá optar quien quiera participar económicamente. Es importante ofrecer un amplio abanico de recompensas, para todos los bolsillos. También hay que establecer el objetivo de financiación, por lo que es necesario llevar a cabo un presupuesto de los futuros gastos del proyecto.

Gran parte del éxito de un proyecto reside en su buena difusión. Por ello, es definitivo contar con una planificación previa de difusión del mismo y realizar un calendario estructurado de acciones relacionadas. Hay que elegir bien al público objetivo y saber cuales son los mejores canales para llegar a él. También hay que realizar un seguimiento diario y continuado de todas las acciones comunicativas.

Los principales motivos por los que los mecenas españoles financian proyectos vía CF son: contribuir a crear lo que me gusta, apoyo directo a los creadores sin intermediarios y contribuir a crear cosas innovadoras. Además la decisión del mecenas de la cantidad aportada está en función de los que recibe a cambio, de las recompensas.

\section{BIBLIOGRAFÍA}

ÁLVAREZ MONZONCILLO, José María y López Villanueva, Javier. El Audiovisual Español: evolución en curso. En Bustamante, Enrique y Rueda, Fernando (Coord.): II Informe sobre el estado de la cultura en España 2914: la salida digital (ICE-2014). Madrid, Observatorio de Cultura y Comunicación, Fundación Alternativas, 2014, pp 65-74.

ASOCIACIÓN ESPAÑOLA DE CROWFUNDING (SCF). Informe de la encuesta de la Asociación Española de Crowdfunding sobre plataformas española de Crowfunding, Rivas- Vaciamadrid (Madrid), SCF, 2014, 14 páginas.

ASOCIACIÓN PARA LA INVESTIGACIÓN DE MEDIOS DE COMUNICACIÓN (AIMC). Estudio General de Medios, ( $3^{a}$ ola) Febrero a Noviembre de 2013 Resumen General. Madrid, AIMC, 2014, 17 páginas.

EUROPEAN COMMISSION. Crowfunding: untapping its potential; reducing the risks. Inputs from the workshop hosted by the European Commission on 3 june 2013. Brussels, European Commission, 2013, 7 páginas. 
BARRAL, Luis Miguel y BARRAL, Pepa. ¿Por qué la gente financia proyectos vía crowfunding? Investigación realizada entre la Comunidad de Mecenas de Lánzanos. Madrid, Two much Research studio, 2014, 123 páginas.

BUSTAMANTE, Enrique. España: La cultura en tiempos de crisis. Fuentes financieras y políticas públicas. Madrid, Observatorio de Cultura y Comunicación, Fundación Alternativas, 2013, 48 páginas.

DOYLE, Gillian. "Economía audiovisual: los mercados audiovisuales en la Unión Europea”, Quaderns del CAC, 2012, vol XV, pp. 15-24.

INTERACTIVE ADVERTISING BUREAU (IAB). IV Estudio anual de Redes sociales. Madrid, IAB, 2013, 58 páginas.

INSTITUTO DE LA CINEMATOGRAFÍA Y DE LAS ARTES AUDIOVISUALES (ICAA). Boletín Informativo de Cine. Año 2013. Madrid, Ministerio de Educación, Cultura y Deporte, ICAA, 2014, 161 páginas.

LAMBERT, Thomas y SCHWIENBACHER, Armin. An Empirical Analysis of Crowdfunding. Louvain. Documento de Trabajo de la Louvain School of Management (Université Catholique de Louvain), 2010, 23 páginas.

ROIG, Antoni. "La participación como bien de consumo: una aproximación conceptual a las formas de implicación de los usuarios en proyectos audiovisuales colaborativos”, Anàlisi, 2010, 40, pp. 101-114.

WARDROP, Robert; ZHANG, Bryan; RAGHAVENDRA, Rau y GRAY, Mia. Moving Mainsttream. The European Alternative Finance Benchmarking Report. Cambridge, University of Cambridge, Cambridge Centre for Alternative Finance, 44 páginas. 